\title{
Zerumbone-loaded nanostructured lipid carrier induces apoptosis in human colorectal adenocarcinoma (Caco2) cell line
}

\begin{abstract}
The incorporation of zerumbone (Zer) into nanostructured lipid carrier (NLC) is hypothesized to increase the efficacy of the drug. Nanostructured lipid carrier has sustained-drug release characteristics and is able to improve the solubility and bioavailability of the lipophilic drug. In this study, the anti-cancer effect of Zer was tested on human colorectal adenocarcinoma (Caco-2) cell line. The effect of Zer, zerumbone-loaded nanostructured lipid carrier (ZerNLC) and NLC on the Caco-2 cell viability were determined using the MTT assay. The treatment concentration ranges from 0 to $120 \mu \mathrm{M}$ at four different time intervals (i.e., $0 \mathrm{~h}, 24$ $\mathrm{hrs}, 48 \mathrm{hrs}$ and $72 \mathrm{hrs}$ ) were evaluated. At $24 \mathrm{hrs}$, the half-growth inhibitory concentration (GI50) of Zer-NLC (i.e., $4.25 \mu \mathrm{M}$ ) is lower than that of Zer (i.e., $23.75 \mu \mathrm{M}$ ). However, Zer outperformed the Zer-NLC at the subsequent time points. Similar trend was observed in other parameters including the cytostatic concentration (CC) and half-lethal concentration 50 (LC50). Phase contrast imaging and AO/PI fluorescence staining were performed at the CC and LC50 values. The morphological changes and the apoptosis features could be seen in cells treated with Zer and Zer-NLC while cells treated with NLC showed minor morphological changes. The cells treated with Zer-NLC demonstrated a slightly slower progression of apoptosis, which could be due to the controlled release of Zer from the NLC matrix. It was concluded that the incorporation of Zer into NLC did not compromise the potency and efficacy of the drug.
\end{abstract}

Keyword: Anti-cancer; Apoptosis; Caco-2; Lipid nanoparticles; Ginger 